\title{
Eosinophil-to-Monocyte Ratio is a Potential Predictor of Prognosis in Acute Ischemic Stroke Patients After Intravenous Thrombolysis
}

\author{
Yueping Chen ${ }^{1, *}$ \\ Junli Ren ${ }^{2,3, *}$ \\ Naiping Yang $2,3, *$ \\ Honghao Huang ${ }^{2,3}$ \\ Xueting $\mathrm{Hu}^{2,3}$ \\ Fangyue Sun $^{2,3}$ \\ Tian Zeng ${ }^{2,3}$ \\ Xinbo Zhou ${ }^{2,3}$ \\ Wenjing $\operatorname{Pan}^{2,3}$ \\ Jingyu $\mathrm{Hu}^{2,3}$ \\ Beibei $\mathrm{Gao}^{4}$ \\ Shunkai Zhang ${ }^{2}$ \\ Guangyong Chen (D) ${ }^{2}$ \\ 'Clinical Laboratory, The Third Affiliated \\ Hospital of Wenzhou Medical University, \\ Wenzhou, People's Republic of China; \\ ${ }^{2}$ Department of Neurology, The Third \\ Affiliated Hospital of Wenzhou Medical \\ University, Wenzhou, People's Republic \\ of China; ${ }^{3}$ School of the First Clinical \\ Medical Sciences, Wenzhou Medical \\ University, Wenzhou, People's Republic \\ of China; ${ }^{4}$ Department of Internal \\ Medicine, The Third Affiliated Hospital of \\ Wenzhou Medical University, Wenzhou, \\ People's Republic of China
}

*These authors contributed equally to this work.

Correspondence: Shunkai Zhang; Guangyong Chen

Department of Neurology, The Third Affiliated Hospital of Wenzhou Medical University, No. 108 Wansong Road, Wenzhou, 325000, Zhejiang, People's

Republic of China

Email shunkaizhang@।26.com;

gychen6@I26.com
Background: Eosinophil and monocyte have been demonstrated separately to be independent predictors of acute ischemic stroke (AIS). This study aimed to evaluate the association between eosinophil-to-monocyte ratio (EMR) and 3-month clinical outcome after treatment with recombinant tissue plasminogen activator (rt-PA) for AIS patients. Simultaneously, we made a simple comparison with other prognostic indicators, such as $24 \mathrm{~h}$ neutrophil-tolymphocyte ratio (NLR) and $24 \mathrm{~h}$ platelet-to-lymphocyte ratio (PLR) to investigate the prognostic value of EMR.

Methods and Results: A total of 280 AIS patients receiving intravenous thrombolysis were retrospectively recruited for this study. Complete blood count evaluations for EMR were conducted on 24 hours admission. The poor outcome at 3 -month was defined as the modified Rankin Scale (mRS) of 3-6 and the mRS score for death was 6. The EMR levels in patients with AIS were lower than those in the healthy controls and showed a negative correlation with the NIHSS score. At the 3-month follow-up, multivariate logistic regression analysis indicated an association among EMR, poor outcome and mortality. In addition, EMR had a higher predictive ability than popular biomarkers like NLR and PLR for 3-month mortality. Conclusion: The lower levels of EMR were independently associated with poor outcome and dead status in AIS patients.

Keywords: acute ischemic stroke, eosinophil-to-monocyte ratio, intravenous thrombolysis, treatment outcome, modified rankin scale

\section{Introduction}

Acute ischemic stroke (AIS) possesses a high morbidity and mortality. And its pathophysiology, an inflammatory process involving endothelial activation, bloodbrain barrier disruption, oxidant and inflammatory mediator accumulation, contributes to brain injury. ${ }^{1}$ Leukocytes, such as neutrophils, monocytes and lymphocytes, are vital inflammatory cells and play essential roles in atherosclerosis development and progression. Ratio indices, calculated by subtypes of white blood cells, such as neutrophil-to-lymphocyte ratio (NLR) and platelet-tolymphocyte ratio (PLR), can be easily performed in routine practice as well as have strong prognostic effects on clinical outcomes of AIS patients. ${ }^{2,3}$ Consequently, there is a growing trend for ratio indices to be used in predictions of thrombolytic outcome in AIS patients. Some reports indicated that eosinophils were inflammatory cells and were associated with severity of AIS. ${ }^{4}$ It has been reported that eosinophil-to-monocyte ratio (EMR) is a popular research topic for 
inflammatory hematologic scores and has an association with mortality in patients with ST-elevation myocardial infarction undergoing primary percutaneous coronary intervention. ${ }^{5,6}$ Both myocardial infarction and AIS are vascular obstructive diseases, which means EMR may possibly connect with the clinical outcome of AIS patients. However, the association between EMR and the outcome of AIS patients receiving thrombolysis has not been fully clarified. Therefore, the purpose of this article was to explore the relationship between EMR and 3-month clinical outcome after thrombolytic therapy with recombinant tissue plasminogen activator (rt-PA) for AIS patients.

\section{Methods}

\section{Study Subjects}

As shown in Figure 1, a total of 486 consecutive patients with a clinical diagnosis of AIS from 2016 to 2019 were retrospectively recruited, in which 76 received a bridging therapy consisting of rt-PA followed by endovascular therapy. They were excluded for the following exclusion criteria: (1) with rheumatic immune diseases; (2) with malignant tumor; (3) with acute myocardial infarction; (4) with the incomplete data [laboratory data such as eosinophil counts and monocyte counts, evaluation data such as modified Rankin Scale (mRS)]; (5) with severe liver damage; (6) with severe kidney damage;

(7) with chronic inflammatory diseases. Finally, 280 cases were included in this analysis. Meanwhile, after propensity score matching, 130 cases were included as healthy controls (HCs).

\section{Data Collection}

Data of patients was collected through the illness record: demographics, medical history (hypertension, diabetes mellitus, hyperlipidemia and atrial fibrillation) and National Institutes of Health Stroke Scale (NIHSS) on admission. Additionally, 3-month mRS after the onset of AIS, collected by two trained physicians on phone interview, was used to

consecutive 486 patients with a clinical diagnosis of AIS who were treated with intravenous thrombolysis from 2016 to 2019

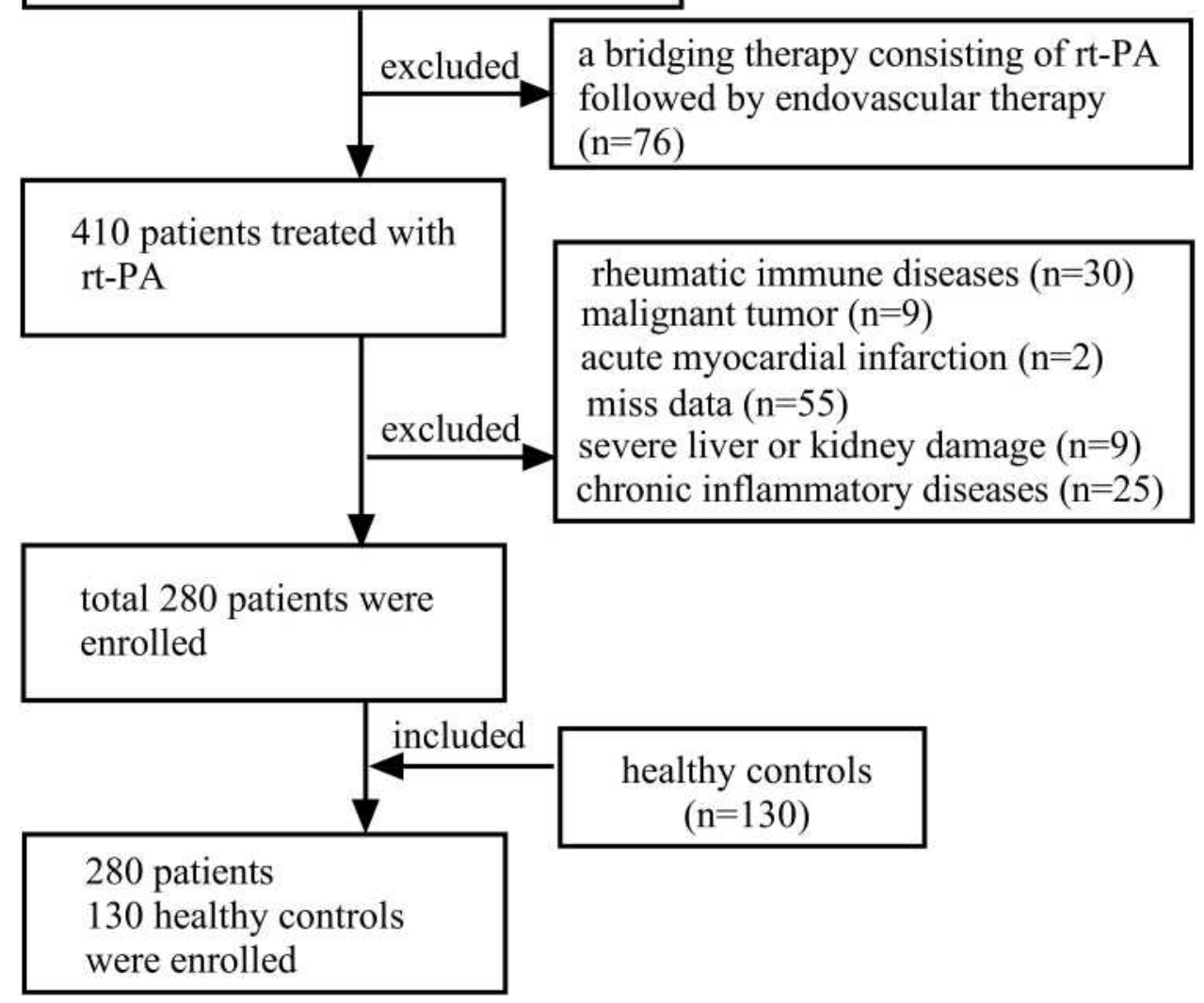

Figure I Flow diagram showing the patient selection process. 
evaluate the functional outcome. Moreover, the variables related to clinical outcomes such as Trial of Org 10,172 in Acute Stroke Treatment (TOAST), infarct volume, Alberta Stroke Program Early CT Score (ASPECT), symptomatic intracranial hemorrhage (sICH), door to needle time (DNT) and onset of needle time (ONT) were also collected. ASPECTS was rated on a scale from 10 (no early signs of ischemia) to 0 (early ischemic changes in all 10 regions). One point was subtracted from 10 for each of the defined regions. ${ }^{7}$ SICH was defined as evidence of hemorrhage on CT or MRI that seemed to be associated with an increase in NIHSS score of $\geq 4 .{ }^{8}$

\section{Assessment of Eosinophil-to-Monocyte Ratio}

The whole blood samples were collected on 24 hours admission and white blood cell counts and peripheral differential counts were measured. EMR was calculated using eosinophil counts divided by monocyte counts. According to the EMR on 24 hours admission, all patients were divided into tertiles.

\section{Definitions of Outcome and Severity}

With respect to NIHSS scores on admission, which could evaluate the severity of AIS, AIS patients were grouped as three levels: mild stroke (NIHSS scores: 0-5), moderate stroke (NIHSS scores: 6-10), severe stroke (NIHSS scores: $>10$ ). ${ }^{9}$ In accordance with 3-month mRS score, all patients were grouped as two levels: good outcome group (mRS scores $\leq 2$ ) and poor outcome group (mRS scores $\geq 3$ ). ${ }^{10}$ In addition, AIS patients with a 3-month mRS score of 3-5 represented major disability while $\mathrm{mRS}=6$ represented death.

\section{Statistical Analysis}

Statistical analyses were performed via SPSS Statistics 24.0 software (SPSS Inc., Chicago, IL) and MedCalc Statistical Software version 15.2.2 (MedCalc Software bvba, Ostend, Belgium; http://www.medcalc.org; 2015). The normality of distribution was evaluated by the Kolmogorov-Smirnov test. Continuous variables were expressed as the mean with standard deviation (mean $\pm \mathrm{SD}$ ) or medians and interquartile range (median, IQR) while categorical variables were described as counts and percentages. Independent sample $t$ test, MannWhitney $U$-test, one-way analysis of variance (ANOVA) or Kruskal-Wallis test were used appropriately for the comparison of continuous data. For categorical variables, chi-square test was performed. The Fisher exact test was used if the expected frequency was equal to or less than 5 . To compare whether there were differences in EMR between HCs and AIS patients, people were divided into AIS group and HCs group. The propensity score matching was used to match the age and sex between the two groups with a match tolerance set at 0.02 . Baseline characteristics according to EMR tertiles and AIS outcomes were also displayed. The Spearman and Chi-square tests were used to analyze the correlation between EMR levels and the severity of AIS. In order to investigate the association between EMR and AIS outcomes, univariate logistic regression analysis was used. In the case where the univariable analysis achieved statistical significance $(p<0.05)$, multivariable test was performed in addition. The receiver operating curve (ROC) was applied to analyze the accuracy of the prognosis of 24h EMR, 24h NLR, and 24h PLR for the 3-month outcome of AIS patients receiving thrombolysis. The differences in discriminative ability were tested using the DeLong method. ${ }^{11}$ Statistical significance was set at $p<0.05$.

The study was approved by the Ethics Committee of our hospital and was performed in accordance with the Declaration of Helsinki. All subjects had signed a written informed consent form.

\section{Results}

\section{Baseline Characteristics of the Study Subjects}

The demographic characteristics and EMR levels of all enrolled subjects are shown in Table 1. After matching of age and sex, the EMR levels in the patients with AIS

Table I Demographic and Laboratory Characteristics of AIS Patients and Healthy Controls

\begin{tabular}{|l|l|l|l|}
\hline \multirow{2}{*}{ Variables } & \multicolumn{2}{l|}{ After Propensity Score Matching } & $\boldsymbol{p}$ value \\
\cline { 2 - 4 } & AIS (n = 130) & HCs (n = 130) & 0.967 \\
Age (years) & $57.50(51.00-65.00)$ & $58.00(51.75-65.00)$ & 0.891 \\
Sex (male, n.\%) & $93(71.5)$ & $92(70.7)$ & $<0.001$ \\
EMR & $0.16(0.06-0.27)$ & $0.27(0.17-0.47)$ & \\
\hline
\end{tabular}

Abbreviations: AIS, acute ischemic stroke; HCs, healthy controls; EMR, eosinophil to monocyte ratio. 
were lower than those in the HCs $(0.16[0.06-0.27]$ vs 0.27 [0.17-0.47]; $p<0.001$ ).

According to EMR levels on 24 hours admission, patients were divided into tertiles as follows: $\mathrm{T} 1 \mathrm{(n}=$ 96): $\mathrm{EMR}<0.09$, T2 $(\mathrm{n}=92): 0.09 \leq \mathrm{EMR} \leq 0.21$ and T3 $(n=92)$ : EMR $>0.21$. Among these three groups, there were no statistical differences in terms of age, sex, hypertension, diabetes, hyperlipidemia, DNT, ONT, sICH, uric acid (UA), urea, creatinine, triglyceride (TC), high-density lipoprotein-cholesterol (HDL-C) and apolipoprotein-A1 (Apo-A1). Besides, proportion of atrial fibrillation, infarct volume, ASPECT, TOAST subtype, white blood cell count (WBC), NLR, and PLR were declined ( $p=0.022, p<$ 0.001, $p<0.001, p=0.047, p<0.001, p<0.001, p<$ 0.001 , respectively) (Table 2 ).

The baseline characteristics of the included patients according to the 3-month outcome are summarized in
Table 3. Among all enrolled 280 patients with AIS, 86 (30.7\%) patients had poor outcome, and 27 (9.6\%) patients had died. Patients with poor outcome exhibited significantly higher age, atrial fibrillation, infarct volume, initial NIHSS scores, WBC, urea, NLR, PLR and significantly lower eosinophil count and EMR level compared to patients with good outcome. Besides, patients died within 3-month follow-up exhibited significantly higher age, atrial fibrillation, initial NIHSS scores, infarct volume, WBC, NLR, PLR and significantly lower eosinophil count, monocyte count, platelet count and EMR levels compared to survivals.

\section{Association Between EMR Levels and the Severity of AIS}

The severity of AIS patients was assessed by the NIHSS score on admission. According to the common definition,

Table 2 Characteristics of AIS Patients According to EMR Tertiles

\begin{tabular}{|c|c|c|c|c|c|}
\hline Variable & Total $(n=280)$ & $E M R<0.09(n=96)$ & $0.09 \leq E M R \leq 0.21(n=92)$ & $E M R>0.21(n=92)$ & p value \\
\hline \multicolumn{6}{|l|}{ Demographic data } \\
\hline Age (years) & $69.00(59.00-77.00)$ & $66.00(60.00-79.75)$ & $70.00(57.25-76.00)$ & $70.00(58.25-77.75)$ & 0.936 \\
\hline Sex (male, n.\%) & $179(63.90)$ & $55(57.20)$ & $62(67.30)$ & $62(67.30)$ & 0.248 \\
\hline \multicolumn{6}{|l|}{ Stroke risk factors (n.\%) } \\
\hline Hypertension (n.\%) & $172(61.40)$ & $58(60.40)$ & $58(63.00)$ & $56(60.80)$ & 0.945 \\
\hline Diabetes (n.\%) & $55(19.60)$ & $15(15.60)$ & $18(19.50)$ & $22(23.90)$ & 0.377 \\
\hline Hyperlipidemia (n.\%) & $32(11.40)$ & $10(10.40)$ & $13(14.10)$ & $9(9.70)$ & 0.604 \\
\hline Atrial fibrillation (n.\%) & $66(23.50)$ & $31(32.20)$ & $21(22.80)$ & $14(15.20)$ & 0.022 \\
\hline DNT (min) & $62.56 \pm 27.51$ & $67.94 \pm 31.01$ & $58.53 \pm 22.87$ & $60.92 \pm 27.24$ & 0.051 \\
\hline ONT (min) & $161.96 \pm 50.18$ & $167.50 \pm 50.48$ & $157.51 \pm 46.95$ & $160.64 \pm 52.95$ & 0.377 \\
\hline Infarct volume $(\mathrm{mL})$ & $4.44(1.37-20.48)$ & $15.04(2.08-46.66)$ & $7.99(1.19-20.91)$ & $2.24(0.50-8.01)$ & $<0.001$ \\
\hline ASPECT & $10(9-10)$ & $9.5(9-10)$ & $10(9-10)$ & $10(10-10)$ & $<0.001$ \\
\hline $\mathrm{sICH}$ & $6(2.14)$ & $4(4.17)$ & I (I.09) & I (1.09) & 0.534 \\
\hline \multicolumn{6}{|l|}{ TOAST subtype (n.\%) } \\
\hline Cardioembolism (n.\%) & $94(33.57)$ & $45(46.88)$ & $25(27.17)$ & $24(26.09)$ & 0.047 \\
\hline Large artery atherosclerosis (n.\%) & $113(40.36)$ & $32(33.33)$ & $39(42.39)$ & $42(45.65)$ & \\
\hline Small vessel occlusion (n.\%) & $40(14.29)$ & $9(9.38)$ & $15(16.30)$ & $16(17.39)$ & \\
\hline Other or undetermined (n.\%) & $33(11.79)$ & $10(10.42)$ & $13(14.13)$ & $10(10.87)$ & \\
\hline \multicolumn{6}{|l|}{ Laboratory data on 24 hours admission } \\
\hline WBC $\left(\times 10^{9} / L\right)$ & $7.40(6.10-8.80)$ & $8.35(7.30-10.78)$ & $7.25(5.83-8.58)$ & $6.60(5.70-7.70)$ & $<0.001$ \\
\hline NLR & $3.23(2.20-5.00)$ & $5.26(3.76-8.29)$ & $2.81(2.07-3.93)$ & $2.56(1.89-3.44)$ & $<0.001$ \\
\hline PLR & $125.80(96.16-169.35)$ & $153.25(116.20-199.04)$ & I I $2.27(87.92-154.58)$ & I $16.75(86.88-152.98)$ & $<0.001$ \\
\hline UA $(\mu \mathrm{mol} / \mathrm{L})$ & $326.66 \pm 85.34$ & $321.67 \pm 88.60$ & $337.23 \pm 82.91$ & $321.03 \pm 84.49$ & 0.368 \\
\hline Urea & $4.83(4.03-6.00)$ & $4.90(4.17-6.24)$ & $5.11(4.20-6.00)$ & $4.58(3.80-5.64)$ & 0.118 \\
\hline Creatinine & $68.00(61.25-76.00)$ & $68.00(61.00-75.25)$ & $68.00(62.00-78.00)$ & $68.00(61.00-75.00)$ & 0.860 \\
\hline $\mathrm{TC}$ & $1.24(0.94-1.65)$ & $1.13(0.86-1.66)$ & $1.27(0.95-1.76)$ & $1.32(0.99-1.65)$ & 0.232 \\
\hline $\mathrm{HDL}-\mathrm{C}(\mathrm{mmol} / \mathrm{L})$ & $1.07(0.93-1.29)$ & $1.09(0.93-1.32)$ & $1.08(0.95-1.30)$ & $1.04(0.92-1.23)$ & 0.498 \\
\hline Apo-Al (g/L) & $1.28(1.15-1.44)$ & $1.29(1.15-1.46)$ & $1.27(1.14-1.48)$ & $1.29(1.18-1.39)$ & 0.931 \\
\hline
\end{tabular}

Abbreviations: AIS, acute ischemic stroke; DNT, door to needle time; ONT, onset to needle time; ASPECT, Alberta Stroke Program Early CT Score; sICH, symptomatic intracranial hemorrhage; TOAST, Trial of Org 10,172 in Acute Stroke Treatment; EMR, eosinophil to monocyte ratio; WBC, white blood cell count; NLR, neutrophil to lymphocyte ratio; PLR, platelet to lymphocyte ratio; UA, uric acid; TC, triglyceride; HDL-C, high density lipoprotein-cholesterol; Apo-AI, apolipoprotein-AI. 
Table 3 Comparison of Clinical Characteristics Between Good and Poor Outcome (or Alive and Dead)

\begin{tabular}{|c|c|c|c|c|c|c|}
\hline & $\begin{array}{l}\text { Good Outcome }(n= \\
194)\end{array}$ & $\begin{array}{l}\text { Poor Outcome }(n= \\
\text { 86) }\end{array}$ & $p$ value & Alive $(n=253)$ & Dead $(n=27)$ & $p$ value \\
\hline \multicolumn{7}{|l|}{ Demographic data } \\
\hline Age (years) & $66.00(56.00-75.00)$ & $75.00(64.00-82.0)$ & $<0.001$ & $68.00(57.50-76.00)$ & $81.00(72.00-85.00)$ & $<0.001$ \\
\hline Sex (male, n.\%) & $129(66.40)$ & $50(58.10)$ & 0.179 & $165(65.20)$ & $14(51.80)$ & 0.169 \\
\hline \multicolumn{7}{|l|}{ Stroke risk factors (n.\%) } \\
\hline Hypertension & II $5(59.20)$ & $57(66.20)$ & 0.219 & $156(61.60)$ & $16(59.20)$ & 0.990 \\
\hline Diabetes & $40(20.60)$ & $15(17.40)$ & 0.566 & $53(20.90)$ & $2(7.40)$ & 0.106 \\
\hline Hyperlipidemia & $18(9.20)$ & $14(16.20)$ & 0.089 & $30(11.80)$ & $2(7.40)$ & 0.751 \\
\hline Atrial fibrillation & $35(18.00)$ & $31(36.00)$ & 0.001 & $53(20.90)$ & $13(48.10)$ & 0.002 \\
\hline DNT (min) & $62.94 \pm 29.34$ & $61.69 \pm 23.02$ & 0.725 & $62.53 \pm 28.29$ & $62.81 \pm 19.14$ & 0.959 \\
\hline ONT (min) & $161.19 \pm 51.09$ & $163.72 \pm 48.34$ & 0.697 & $160.64 \pm 50.54$ & $174.33 \pm 45.73$ & 0.178 \\
\hline Infarct volume (mL) & $2.68(0.92-12.65)$ & $21.51(3.99-66.79)$ & $<0.001$ & $4.11(1.26-17.92)$ & $63.50(28.82-131.47)$ & 0.001 \\
\hline ASPECT & $10(9-10)$ & $9(8-10)$ & $<0.001$ & $10(9-10)$ & $9(8-10)$ & 0.001 \\
\hline $\mathrm{slCH}$ & 0 & $6(7.00)$ & 0.001 & $2(0.79)$ & $4(14.81)$ & 0.001 \\
\hline \multicolumn{7}{|l|}{ Evaluation of stroke } \\
\hline NIHSS on admission & $6(4.00-9.00)$ & $13(8.00-18.50)$ & $<0.001$ & $6(4-10)$ & $18(13.00-21.00)$ & $<0.001$ \\
\hline 3-month mRS & $I(0-1)$ & $4(3-6)$ & $<0.001$ & I (0-2) & $6(6)$ & $<0.001$ \\
\hline \multicolumn{7}{|l|}{ TOAST subtype (n.\%) } \\
\hline Cardioembolism & $48(24.74)$ & $46(53.49)$ & $<0.001$ & $72(28.46)$ & $22(81.48)$ & $<0.001$ \\
\hline Large artery atherosclerosis & $83(42.78)$ & $30(34.88)$ & & $110(43.48)$ & $3(11.11)$ & \\
\hline Small vessel occlusion & $40(20.62)$ & 0 & & $40(15.81)$ & 0 & \\
\hline Other or undetermined & $23(11.86)$ & $10(11.63)$ & & $31(12.25)$ & $2(7.4 I)$ & \\
\hline \multicolumn{7}{|l|}{$\begin{array}{l}\text { Laboratory data on } 24 \text { hours } \\
\text { admission }\end{array}$} \\
\hline WBC $\left(\times 10^{9} / \mathrm{L}\right)$ & $7.10(5.70-8.20)$ & $8.55(7.20-10.63)$ & $<0.001$ & $7.30(6.00-8.70)$ & $8.70(7.30-11.50)$ & 0.004 \\
\hline Eosinophil $\left(\times 10^{9} / \mathrm{L}\right)$ & $0.09(0.04-0.15)$ & $0.03(0.01-0.08)$ & $<0.001$ & $0.08(0.03-0.14)$ & $0.01(0.00-0.02)$ & $<0.001$ \\
\hline Monocyte $\left(\times 10^{9} / \mathrm{L}\right)$ & $0.52 \pm 0.25$ & $0.50 \pm 0.21$ & 0.597 & $0.50(0.40-0.60)$ & $0.40(0.30-0.60)$ & 0.021 \\
\hline Platelet count $\left(\times 10^{9} / \mathrm{L}\right)$ & $196.49 \pm 54.02$ & $190.88 \pm 58.85$ & 0.437 & $197.68 \pm 55.09$ & $167.48 \pm 52.69$ & 0.007 \\
\hline $\mathrm{UA}(\mu \mathrm{mol} / \mathrm{L})$ & $330.82 \pm 81.51$ & $315.59 \pm 94.50$ & 0.200 & $324.44 \pm 82.89$ & $354.89 \pm 110.82$ & 0.134 \\
\hline Urea & $4.70(3.96-5.63)$ & $5.60(4.27-6.30)$ & 0.001 & $4.79(4.03-5.94)$ & $5.49(4.12-6.37)$ & 0.197 \\
\hline Creatinine & $68.00(60.50-75.50)$ & $68.00(63.00-78.00)$ & 0.327 & $68.00(62.00-76.00)$ & $68.00(61.00-88.00)$ & 0.418 \\
\hline $\mathrm{TC}$ & $1.29(0.96-1.65)$ & $1.16(0.90-1.67)$ & 0.232 & $1.24(0.94-1.65)$ & $1.29(0.99-1.86)$ & 0.661 \\
\hline $\mathrm{HDL}-\mathrm{C}(\mathrm{mmol} / \mathrm{L})$ & $1.07(0.95-1.28)$ & $1.07(0.91-1.33)$ & 0.921 & $1.07(0.94-1.29)$ & $1.02(0.81-1.32)$ & 0.237 \\
\hline Apo-Al (g/L) & $1.30(1.17-1.43)$ & $1.23(1.12-1.48)$ & 0.282 & $1.28(1.15-1.44)$ & $1.25(1.02-1.47)$ & 0.498 \\
\hline EMR & $0.18(0.10-0.29)$ & $0.05(0.02-0.15)$ & $<0.001$ & $0.16(0.08-0.28)$ & $0.02(0.00-0.04)$ & $<0.001$ \\
\hline NLR & $2.77(2.04-3.96)$ & $4.44(3.49-7.80)$ & $<0.001$ & $3.00(2.15-4.42)$ & $6.38(4.00-11.00)$ & $<0.001$ \\
\hline PLR & $121.30(91.59-162.93)$ & $143.46(106.4 \mid-192.04)$ & 0.011 & 125.00 & $153.64(100.00-242.86)$ & 0.042 \\
\hline
\end{tabular}

Abbreviations: AIS, acute ischemic stroke; DNT, door to needle time; ONT, onset to needle time; ASPECT, Alberta Stroke Program Early CT Score; sICH, symptomatic intracranial hemorrhage; TOAST, Trial of Org 10,172 in Acute Stroke Treatment; NIHSS, national institute of health stroke scale; mRS, modified Rankin Scale; WBC, white blood cell count; UA, uric acid; TC, triglyceride; HDL-C, high density lipoprotein-cholesterol; Apo-AI, apolipoprotein-AI; EMR, eosinophil to monocyte ratio; NLR, neutrophil to lymphocyte ratio; PLR, platelet to lymphocyte ratio.

AIS patients were divided into mild stroke group (NIHSS scores: $0-5, \mathrm{n}=101$ ), moderate stroke group (NIHSS scores: $6-10, \mathrm{n}=100)$ and severe stroke group (NIHSS scores: $>10$, $\mathrm{n}=79$ ). We found that the patients with lower EMR levels were more likely to have moderate or severe stroke $(p<$ $0.001)$ and severe stroke $(p<0.001)$ (Figure 2A).

\section{Association Between EMR Levels and the 3-Month Outcome}

The distribution of mRS scores in the three EMR groups is shown in Figure 2B. Up to $53.1 \%$ of patients developed poor outcome and up to $26.0 \%$ of patients died in the low EMR levels group (T1: EMR < 0.09). Proportion of poor 

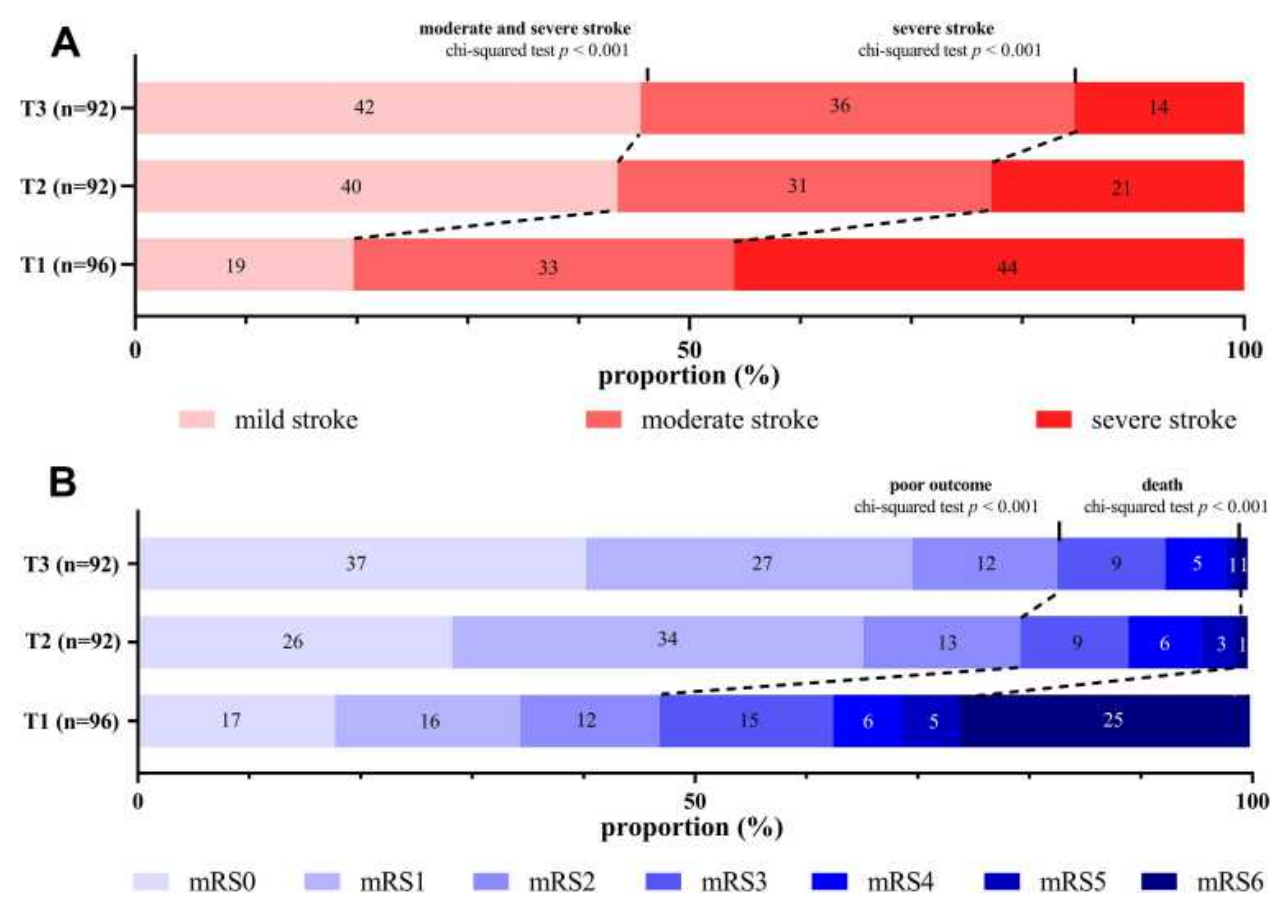

Figure 2 (A) Distribution of NIHSS on admission in the tertiles of increasing EMR levels. (B) Distribution of 3-month mRS scores in the tertiles of increasing EMR levels.

outcome and death was $20.6 \%$ and $1.0 \%$ in the moderate EMR levels group (T2: $0.09 \leq \mathrm{EMR} \leq 0.21$ ). Besides, the proportion of poor outcome and death was $17.3 \%$ and $1.0 \%$ in the high EMR group (T3: EMR > 0.21). Patients were more likely to have a worse outcome if they had lower EMR.

Univariate logistic regression analysis demonstrated that the age, atrial fibrillation, $\mathrm{WBC}, \mathrm{EMR}^{\dagger}$ (per 0.01- point increase in EMR) were associated with poor outcome and dead status (Table 4). In model 1, compared to the subjects in $\mathrm{T} 1$, the odds ratio (OR) of subjects in T3 was $0.186(95 \%$ CI $0.095-0.364, p<0.001)$ for poor outcome and 0.031 (95\% CI 0.004-0.236, $p=0.001)$ for death. After adjusting for age (Model 2), the odds ratio (OR) of the subjects in T3 was 0.153 (95\% CI $0.074-$ $0.314, p<0.001)$ for poor outcome and 0.027 (95\% CI

Table 4 Univariate Logistic Regression Analyses for Prognosis

\begin{tabular}{|c|c|c|c|c|}
\hline \multirow[t]{2}{*}{ Variables } & \multicolumn{4}{|l|}{ Univariate Logistic Regression } \\
\hline & Poor Outcome OR $(95 \% \mathrm{CI})$ & $p$ value & Dead OR (95\% Cl) & $p$ value \\
\hline Age (years) & $1.057(1.032-1.083)$ & $<0.001$ & $1.080(1.036-1.126)$ & $<0.001$ \\
\hline Sex (male, n.\%) & $0.700(0.4|5-| . \mid 79)$ & 0.180 & $0.574(0.259-1.276)$ & 0.173 \\
\hline Hypertension (n.\%) & $1.398(0.819-2.389)$ & 0.220 & $0.995(0.434-2.28 I)$ & 0.990 \\
\hline Diabetes (n.\%) & $0.825(0.428-1.592)$ & 0.566 & $0.314(0.072-1.373)$ & 0.124 \\
\hline Hyperlipidemia (n.\%) & $1.901(0.898-4.026)$ & 0.093 & $0.595(0.134-2.638)$ & 0.494 \\
\hline Atrial fibrillation (n.\%) & $2.56 \mathrm{I}(1.445-4.539)$ & 0.001 & $3.504(1.554-7.903)$ & 0.003 \\
\hline WBC $\left(\times 10^{9} / \mathrm{L}\right)$ & 1.377 (I.220-I.554) & $<0.001$ & 1.290 (I.I II-I.499) & 0.001 \\
\hline $\mathrm{UA}(\mu \mathrm{mol} / \mathrm{L})$ & $0.998(0.995-1.001)$ & 0.200 & $1.004(0.999-1.009)$ & 0.136 \\
\hline Urea & $1.114(0.990-1.255)$ & 0.073 & $1.103(0.993-1.226)$ & 0.068 \\
\hline Creatinine & I.00I (0.998-I.005) & 0.518 & $1.003(0.999-1.007)$ & 0.112 \\
\hline TC & $0.84 \mid(0.620-1.139)$ & 0.263 & $0.952(0.6 \mid 0-1.485)$ & 0.828 \\
\hline HDL-C (mmol/L) & $1.098(0.393-3.063)$ & 0.859 & $0.304(0.044-2.087)$ & 0.226 \\
\hline Apo-AI (g/L) & $0.689(0.214-2.220)$ & 0.532 & $0.324(0.035-2.977)$ & 0.320 \\
\hline $\mathrm{EMR}^{\dagger}$ & $0.970(0.953-0.988)$ & 0.001 & $0.821(0.750-0.898)$ & $<0.001$ \\
\hline
\end{tabular}

Abbreviations: WBC, white blood cell count; UA, uric acid; TC, triglyceride; HDL-C, high density lipoprotein-cholesterol; Apo-AI, apolipoprotein-A I; EMR, eosinophil to monocyte ratio; ${ }^{\dagger} \mathrm{OR}$ is intended for per $0.0 \mathrm{I}$-point increase of EMR. 
0.003-0.206, $p=0.001)$ for death when compared to the subjects in T1. Even after adjusting for all confounders shown in the univariate logistic regression (Model 3), the relationship between EMR and stroke outcome still remained significant in T3 with OR of $0.270(95 \% \mathrm{CI}$ : $0.124-0.589, p=0.001)$ for poor outcome and $0.036(95 \%$ CI: $0.004-0.292, p=0.002$ ) for death (Table 5). In addition, the OR values of T2 and T3 were close so that the relationship between EMR, poor outcome and mortality has appeared to be L-shaped. The result suggested that the lower levels of EMR were independently associated with poor outcome and mortality rate in AIS patients.

\section{Receiver Operating Characteristic (ROC)} Curve Analysis for 3-Month Outcome

ROC curves were performed to differentiate the efficiency of EMR and other popular biomarkers like NLR and PLR in predicting 3-month poor outcome (Figure 3A) and mortality (Figure 3B). There was no significant difference in efficiency between EMR and NLR for predicting poor outcome (AUC 0.730 vs 0.739; $p=0.790$ ). Besides, EMR had a higher accuracy rate in predicting poor outcome than the level of PLR (AUC 0.730 vs 0.595; $p=$ 0.001). The optimal cut-off value of the EMR that best discriminated poor outcome was 0.05 (55.8\% sensitivity and $89.2 \%$ specificity). As for predicting mortality, EMR had a higher accuracy rate than NLR (AUC 0.877 vs $0.810 ; p=0.024$ ) and PLR (AUC 0.877 vs $0.619 ; p<$ $0.001)$. What is more, the optimal cut-off values of the
EMR that best discriminated mortality were $0.05(88.9 \%$ sensitivity and $82.2 \%$ specificity). Moreover, when we combined EMR and NLR, we found that there was also no significant difference in efficiency between them and single EMR in the aspect of predicting poor outcome or mortality (AUC 0.752 vs $0.730, p=0.340 ; 0.882$ vs 0.877 , $p=0.615)($ Table 6$)$.

\section{Discussion}

In this retrospective interventional cohort study, it has been demonstrated that lower EMR level was an independent predictor of 3-month poor outcome for AIS patients receiving thrombolysis. To our knowledge, this is the first study to expound that EMR was related to clinical outcome and death status. The main findings of our study were as follows: (1) Lower EMR level was significantly associated with 3-month poor outcome and death status in AIS patients. (2) ROC curve analysis showed that EMR had a higher accuracy in predicting poor outcome than the level of PLR and a similar predictive ability compared to NLR. As for predicting mortality, EMR had a higher accuracy than NLR and PLR. (3) After adjustments, the predictive value of EMR was still significant.

Previous studies have shown that lower EMR levels are significantly related to the poor prognosis after treatment with rt-PA for AIS patients. ${ }^{12}$ Meanwhile, other researches have suggested that AIS patients with lower EMR levels are more likely to have limb dysfunction and difficult to recover. ${ }^{13}$ AIS is mainly caused by atherosclerosis of the

Table 5 Adjusted Models for Prognosis at 3 Months

\begin{tabular}{|c|c|c|c|c|}
\hline \multirow[t]{2}{*}{ Variables } & \multicolumn{4}{|l|}{ Multiple Logistic Regression } \\
\hline & Poor Outcome OR (95\% Cl) & $p$ value & Dead OR ( $95 \% \mathrm{Cl})$ & $p$ value \\
\hline \multicolumn{5}{|l|}{ Model I } \\
\hline TI & 1 & & I & \\
\hline $\mathrm{T} 2$ & $0.230(0.121-0.438)$ & $<0.001$ & $0.031(0.004-0.236)$ & 0.001 \\
\hline T3 & $0.186(0.095-0.364)$ & $<0.001$ & $0.031(0.004-0.236)$ & 0.001 \\
\hline \multicolumn{5}{|l|}{ Model 2} \\
\hline $\mathrm{TI}$ & 1 & & I & \\
\hline $\mathrm{T} 2$ & $0.197(0.099-0.394)$ & $<0.001$ & $0.027(0.003-0.210)$ & 0.001 \\
\hline T3 & $0.153(0.074-0.314)$ & $<0.001$ & $0.027(0.003-0.206)$ & 0.001 \\
\hline \multicolumn{5}{|l|}{ Model 3} \\
\hline TI & 1 & & I & \\
\hline $\mathrm{T} 2$ & $0.295(0.142-0.610)$ & 0.001 & $0.034(0.004-0.272)$ & 0.001 \\
\hline T3 & $0.270(0.124-0.589)$ & 0.001 & $0.036(0.004-0.292)$ & 0.002 \\
\hline
\end{tabular}

Notes: Model I is univariate analysis. Model 2 is adjusted for age. Model 3 for poor outcome is adjusted for age, atrial fibrillation and white blood cell count. Model 3 for death is adjusted for age, atrial fibrillation and white blood cell count. 

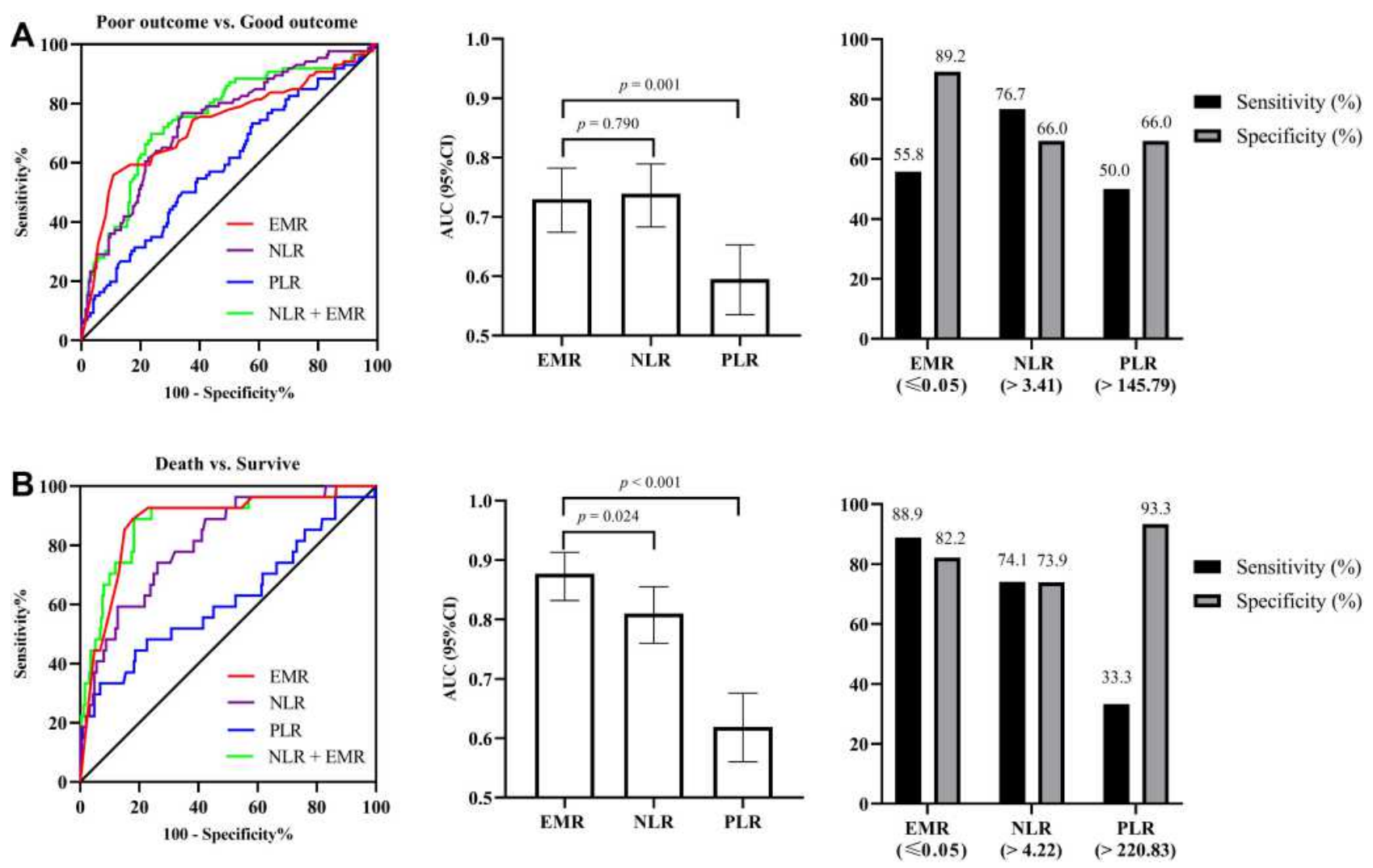

Figure 3 (A) Receiver operating characteristic curve (ROC) of EMR, NLR, PLR and NLR + EMR on the prognosis of AIS patients between poor outcome and good outcome. (B) Receiver operating characteristic curve (ROC) of EMR, NLR, PLR and NLR + EMR on the prognosis of AIS patients between death and survive.

large arteries. The accumulation and infiltration of eosinophils around the cerebral blood vessel walls could cause a series of inflammation and vascular injury reactions as well as aggravate the formation of unstable atherosclerosis of the arteries, which can easily lead to AIS. In addition, some studies indicated that monocytes were associated with the outcome of AIS patients with the following reasons. ${ }^{14,15}$ First, cerebral ischemia and hypoxia would stimulate monocytes to produce inflammatory mediators, such as interleukin-1 (IL-1), IL-6, IL-8 and tumor necrosis factor (TNF). Inflammation further aggravated cerebral ischemia and hypoxia, making brain tissue damage more serious. ${ }^{16}$ Second, monocytes could activate platelets to form platelet-monocyte aggregates (PMAs), which promote the release of inflammatory response factors, adhesion factors and vasoactive substances. PMAs could also

Table 6 Diagnostic Values of the EMR, NLR, PLR and NLR + EMR for Stroke Outcome

\begin{tabular}{|l|l|l|l|l|l|l|}
\hline & AUC (95\% Cl) & $p$ & $p^{*}$ & Cutoff Value & Sensitivity (\%) & Specificity (\%) \\
\hline Poor outcome & & & & & & \\
EMR & $0.730(0.674-0.782)$ & $<0.001$ & Ref & $\leq 0.05$ & 55.8 & 89.2 \\
NLR & $0.739(0.683-0.789)$ & $<0.001$ & 0.790 & $>3.41$ & 76.7 & 66.0 \\
PLR & $0.595(0.535-0.653)$ & 0.011 & 0.001 & $>145.79$ & 50.0 & 66.0 \\
NLR + EMR & $0.752(0.697-0.802)$ & $<0.001$ & 0.340 & $>0.30$ & 69.8 & 76.3 \\
\hline Dead & & & & & & \\
EMR & $0.877(0.832-0.913)$ & $<0.001$ & Ref & $\leq 0.05$ & 88.9 & 73.1 \\
NLR & $0.810(0.760-0.855)$ & $<0.001$ & 0.024 & $>4.22$ & & \\
PLR & $0.619(0.560-0.676)$ & 0.072 & $<0.001$ & $>220.83$ & 33.3 & 93.3 \\
NLR + EMR & $0.882(0.838-0.917)$ & $<0.001$ & 0.615 & $>0.13$ & 88.9 & 81.8 \\
\hline
\end{tabular}

Note: *p for comparison of AUC between groups.

Abbreviations: AUC, area under the curve; Cl, confidence interval; EMR, eosinophil to monocyte ratio; NLR, neutrophil to lymphocyte ratio; PLR, platelet to lymphocyte ratio. 
accelerate thrombosis and vascular occlusion, causing the change in hemodynamics and aggravating cerebral ischemia damage. ${ }^{17,18}$

New indexes, calculated by subtypes of white blood cells, are better than single inflammatory cells in reflecting the systemic inflammation. EMR is a new parameter but the association between EMR and outcome of AIS patients has not been fully clarified. Our study demonstrated that lower EMR levels were related to poor outcome and mortality in AIS patients. We found that the lower EMR levels resulted from the decrease in eosinophil counts while the monocyte counts had no statistical differences among the three groups. In contrast to our current findings, other researchers found that monocyte counts were statistically significant in AIS. ${ }^{14}$ The reason for this discrepancy might be that the sample size they studied was small and the subjects were recruited from different regions. Here, we propose several hypotheses to investigate the causes of eosinophil penia in AIS patients. First, eosinophil apoptosis and degranulation are induced by inflammatory cytokines, resulting in excessive loss of eosinophil. ${ }^{19}$ Patients with larger infarct size may be prone to acute stress responses that stimulate the release of adrenal glucocorticoids and epinephrine, leading to a decrease in eosinophils. ${ }^{20,21}$ Secondly, the release of inflammatory cytokines after cerebral ischemia causes eosinophils to migrate to the inflammatory site, resulting in peripheral blood eosinophil reduction. ${ }^{22}$

Since a single inflammatory cell count has disadvantages in summarizing the overall systemic inflammation, it is a current research hotspot to propose novel indicators through combining different subtypes of white blood cells, like NLR and PLR. Above all, EMR is a potential prognostic marker with its convenience of calculating as eosinophils and monocytes were collected in the blood routine test.

Inevitably, our study has some limitations. First of all, the sample size of our study is relatively diminutive. Furthermore, because the patients participating in this study were from the same hospital, the results we got had certain limitations. Lastly, the mechanism connecting the prognostic effect of EMR on AIS patients and the interactions between eosinophils and monocytes were not fully understood. Therefore, more experimental studies are needed to explain this mechanism. However, our study may provide vital clues to the clinic.

\section{Conclusion}

The lower levels of EMR were independently associated with poor outcome and dead status for AIS patients.

\section{Data Sharing Statement}

The data that support the findings of this study are available from the corresponding author on reasonable request.

\section{Ethics Approval and Consent to Participate}

The study was approved by the Ethics Committee of the Third Affiliated Hospital of Wenzhou Medical University and was performed in accordance with the Declaration of Helsinki. All subjects signed a written informed consent form.

\section{Consent for Publication}

Not applicable.

\section{Acknowledgments}

We sincerely thank the participating hospitals, AIS patients, their families and colleagues who have provided valuable suggestions for this study. Yueping Chen, Junli Ren, and Naiping Yang are co-first authors of this study.

\section{Author Contributions}

Conceptualization and design, SZ and GC; Methodology, SZ, GC, YC and JR; Software, JR and NY; Validation, JR and NY; Formal Analysis, JR, NY, HH and XH; Investigation, SZ and GC; Resources, SZ and GC; Data Curation, YC, JR, NY, HH, XH, FS, TZ, XZ, WP, JH and BG; Writing-Original Draft Preparation, YC, JR, NY; Writing-Review \& Editing, SZ, GC, YC, JR, NY, HH, $\mathrm{XH}, \mathrm{FS}, \mathrm{TZ}, \mathrm{XZ}, \mathrm{WP}, \mathrm{JH}$ and BG; Visualization, YC, JR, and NY; Supervision, SZ and GC; Project Administration, SZ and GC. All authors contributed to data analysis, drafting or revising the article, have agreed on the journal to which the article will be submitted, gave final approval for the version to be published, and agreed to be accountable for all aspects of the work.

\section{Disclosure}

The authors declared no potential conflicts of interest with respect to the research, authorship, and/or publication of this article. 


\section{References}

1. Ishikawa M, Zhang JH, Nanda A, Granger DN. Inflammatory responses to ischemia and reperfusion in the cerebral microcirculation. Front Biosci. 2004;9:1339-1347. doi:10.2741/1330

2. Tokgoz S, Kayrak M, Akpinar Z, Seyithanoğlu A, Güney F, Yürüten B. Neutrophil lymphocyte ratio as a predictor of stroke. J Stroke Cerebrovasc Dis. 2013;22:1169-1174. doi:10.1016/j. jstrokecerebrovasdis.2013.01.011

3. $\mathrm{Xu} \mathrm{JH}, \mathrm{He} \mathrm{XW}, \mathrm{Li} \mathrm{Q}$, et al. Higher platelet-to-lymphocyte ratio is associated with worse outcomes after intravenous thrombolysis in acute ischaemic stroke. Front Neurol. 2019;10:1192. doi:10.3389/ fneur.2019.01192

4. Wang J, Ma L, Lin T, Li SJ, Chen LL, Wang DZ. The significance of eosinophils in predicting the severity of acute ischemic stroke. Oncotarget. 2017;8:104238-104246. doi:10.18632/oncotarget.22199

5. Yucel C, Keskin MZ, Cakmak O, et al. Predictive value of pre-operative inflammation-based prognostic scores (neutrophil-tolymphocyte ratio, platelet-to-lymphocyte ratio, and monocyte-toeosinophil ratio) in testicular sperm extraction: a pilot study. Andrology. 2017;5:1100-1104. doi:10.1111/andr.12417

6. Deng X, Wang X, Shen L, et al. Association of eosinophil-tomonocyte ratio with 1-month and long-term all-cause mortality in patients with st-elevation myocardial infarction undergoing primary percutaneous coronary intervention. $J$ Thorac Dis. 2018;10:5449-5458. doi:10.21037/jtd.2018.09.27

7. Maegerlein C, Fischer J, Mönch S, et al. Automated calculation of the Alberta stroke program early ct score: feasibility and reliability. Radiology. 2019;291:141-148. doi:10.1148/radiol.2019181228

8. Hacke W, Kaste M, Bluhmki E, et al. Thrombolysis with alteplase 3 to 4.5 hours after acute ischemic stroke. $N$ Engl $J$ Med. 2008;359:1317-1329. doi:10.1056/NEJMoa0804656

9. Åsberg S, Farahmand B, Hasvold P, Johansson S, Appelros P. Noncardioembolic tia and ischemic stroke: implications of severity. Acta Neurol Scand. 2018;138:369-376. doi:10.1111/ane.12974

10. Pan H, Fu M, Ge W, Zhou C. The effects of changes in platelet-toneutrophil ratios 24 hours after intravenous thrombolysis on prognosis in acute ischemic stroke patients. Clin Neurol Neurosurg. 2020;190:105739. doi:10.1016/j.clineuro.2020.105739

11. DeLong ER, DeLong DM, Clarke-Pearson DL. Comparing the areas under two or more correlated receiver operating characteristic curves: a nonparametric approach. Biometrics. 1988;44:837-845. doi: $10.2307 / 2531595$
12. Montgomery ND, Dunphy CH, Mooberry M, et al. Diagnostic complexities of eosinophilia. Arch Pathol Lab Med. 2013;137:259-269. doi:10.5858/arpa.2011-0597-RA

13. Weller PF, Spencer LA. Functions of tissue-resident eosinophils. Nat Rev Immunol. 2017;17:746-760. doi:10.1038/nri.2017.95

14. Liberale L, Montecucco F, Bonaventura A, et al. Monocyte count at onset predicts poststroke outcomes during a 90-day follow-up. Eur J Clin Invest. 2017;47:702-710. doi:10.1111/eci.12795

15. Dong X, Nao J, Gao Y. Peripheral monocyte count predicts outcomes in patients with acute ischemic stroke treated with rtpa thrombolysis. Neurotox Res. 2020;37:469-477. doi:10.1007/s12640-019-00103-0

16. Foey AD, Parry SL, Williams LM, Feldmann M, Foxwell BM, Brennan FM. Regulation of monocyte il-10 synthesis by endogenous il-1 and tnf-alpha: role of the p38 and p42/44 mitogen-activated protein kinases. J Immunol. 1998;160:920-928.

17. Konstantopoulos K, Grotta JC, Sills C, Wu KK, Hellums JD. Shearinduced platelet aggregation in normal subjects and stroke patients. Thromb Haemost. 1995;74:1329-1334. doi:10.1055/s-0038-1649935

18. Ray MJ, Walters DL, Bett JN, Cameron J, Wood P, Aroney CN. Platelet-monocyte aggregates predict troponin rise after percutaneous coronary intervention and are inhibited by abciximab. Int $J$ Cardiol. 2005;101:249-255. doi:10.1016/j.ijcard.2004.03.033

19. Cayrol C, Girard JP. Interleukin-33 (il-33): a nuclear cytokine from the il-1 family. Immunol Rev. 2018;281:154-168. doi:10.1111/ imr. 12619

20. Bass DA, Gonwa TA, Szejda P, Cousart MS, DeChatelet LR, McCall CE. Eosinopenia of acute infection: production of eosinopenia by chemotactic factors of acute inflammation. J Clin Invest. 1980;65:1265-1271. doi:10.1172/JCI109789

21. Pazdrak K, Straub C, Maroto R, et al. Cytokine-induced glucocorticoid resistance from eosinophil activation: protein phosphatase 5 modulation of glucocorticoid receptor phosphorylation and signaling. $J$ Immunol. 2016;197:3782-3791. doi:10.4049/ jimmunol.1601029

22. Cowan MJ, Reichenbach D, Turner P, Thostenson C. Cellular response of the evolving myocardial infarction after therapeutic coronary artery reperfusion. Hum Pathol. 1991;22:154-163. doi:10.1016/0046-8177(91)90037-P
Clinical Interventions in Aging

\section{Publish your work in this journal}

Clinical Interventions in Aging is an international, peer-reviewed journal focusing on evidence-based reports on the value or lack thereof of treatments intended to prevent or delay the onset of maladaptive correlates of aging in human beings. This journal is indexed on PubMed Central, MedLine, CAS, Scopus and the Elsevier
Bibliographic databases. The manuscript management system is completely online and includes a very quick and fair peer-review system, which is all easy to use. Visit http://www.dovepress.com/ testimonials.php to read real quotes from published authors. 